\title{
Bone Volumes and Trajectory Angles for Acetabular Anchor Placement Can Be Optimized
}

\author{
Rai Di Loreto, M.Sc., Alan Getgood, M.Phil., M.D., F.R.C.S. (Tr.\&Orth.), Ryan Degen, M.D., \\ and Timothy A. Burkhart, Ph.D.
}

\begin{abstract}
Purpose: The purpose of this study was to determine the optimal anchor placement and trajectory when repairing acetabular labral tears during hip arthroscopy with the primary focus on the 12 to 3 o'clock positions on the acetabular rim. Methods: Three-dimensional computational models of the pelvis were generated from 13 cadaveric specimens using 3D slicer medical imaging software. A set of cones, consistent with the dimensions of a commonly used sutured anchor, were virtually embedded into the models at the 12, 1, 2, and 3 o'clock positions around the acetabulum. Mirror images of the cone were extended toward the superficial aspect of the hip. The volume of bone occupied by the virtual anchor, the trajectory angle, and the volume of overlap between adjacent anchor locations were calculated. Results: Bone volume was significantly greater at the $1 \mathrm{o}^{\prime}$ clock position $\left(4196.2\right.$ [1 190.2] $\left.\mathrm{mm}^{3}\right)$ compared with all other positions $(P<.001)$. The 3 o'clock position had the smallest volume $\left(629.2[180.0] \mathrm{mm}^{3}\right)$ and was also significantly less than the $12(P<.001)$ and 2 $\mathrm{o}^{\prime}$ clock $(P=.014)$ positions $)$. The trajectory angle of $\left.32.04[5.05]^{\circ}\right)$ at the $1 \mathrm{o}^{\prime}$ clock position was significantly greater compared with all other positions $(P<.001)$. The least amount of adjacent position overlap occurred between the 2 and 3 o'clock positions $\left(.12[.42] \mathrm{mm}^{3}\right)$, and this was statistically smaller than the overlap between cones at the 12 and $1 \mathrm{o}^{\prime} \mathrm{clock}$

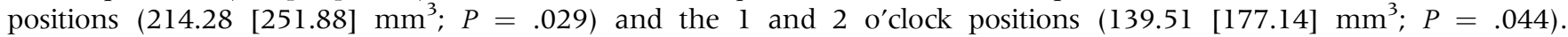
Conclusions: Trajectory angles and the thickness of bone around the acetabulum were the greatest at the 12 to 1 o'clock positions, with the $1 \mathrm{o}^{\prime}$ clock position identified as that with the largest trajectory angle for safe anchor insertion. Clinical Relevance: The use of a single, workhorse portal, for anchor insertion may not be recommended and careful selection of a portal allowing a direct approach should be used for anterior anchor insertion.
\end{abstract}

\section{Introduction}

A rthroscopy hip preservation is an established treatment of labral tears and FAI with successful short-term and mid-term outcomes across the published literature. ${ }^{1}$ From 2004 to 2009, the rate of this procedure increased from 1.2 cases per 10,000 patients

From the School of Kinesiology, Western University, London, Ontario, Canada (R.D.L.); Fowler Kennedy Sports Medicine Clinic, Western University, London, Ontario, Canada (A.G., R.D.); and Kinesiology and Physical Education, University of Toronto, Toronto, Ontario, Canada (T.A.B.).

The authors report the following potential conflicts of interest or sources of funding: A.G.reports grants from Smith $\theta$ Nephew and Ossur; personal fees from Smith $\theta$ Nephew, Precision OS, Xiros, Olympus, and Graymont, outside the submitted work. T.A.B. reports grants from Smith $\theta$ Nephew during the conduct of the study. Full ICMJE author disclosure forms are available for this article online, as supplementary material.

Received April 28, 2021; accepted October 24, 2021.

Address correspondence to Timothy A. Burkhart, Ph.D., Kinesiology and Physical Education, University of Toronto, Toronto, ON, M5S 2W6, Canada. E-mail: timothy.burkhart@utoronto.ca

(C) 2021 THE AUTHORS. Published by Elsevier Inc. on behalf of the Arthroscopy Association of North America. This is an open access article under the CC BY-NC-ND license (http://creativecommons.org/licenses/by-nc-nd/4.0/). 2666-061X/21603

https://doi.org/10.1016/j.asmr.2021.10.022 to 5.6 per 10,000 patients. There was a further $220 \%$ increase from 2014 to $2017 .^{2,3}$ The increasing popularity of hip arthroscopy is partially attributed to the minimally invasive nature of the procedure and the ability to treat a variety of hip pathologies. ${ }^{4-6}$

The anterior-superior region of the acetabular labrum is a commonly injured structure, accounting for approximately $85 \%$ of all labral injuries. As such, a large proportion of arthroscopic procedures focus on this region for labral repair. ${ }^{7,8}$ However, it can be challenging during surgery to safely place suture anchors, and techniques are often adjusted to help identify the safest position and trajectory for anchor insertion. ${ }^{7,9-11}$ The primary goal of the suture anchor insertion is to position them as close to the articular cartilage without penetrating the joint space, to allow anatomic apposition of the labrum along the acetabular rim. ${ }^{5,12-15}$

Using the clock face method, labral tears can be visualized around the acetabulum in order to locate the safest locations for anchor placement, while providing optimal clinical outcomes. ${ }^{16-18}$ A reference point, which denotes the location of a prominent anatomical 
landmark that is consistent across all patients, is needed to orient the clock face. It is widely accepted that the center of the transverse acetabular ligament be used to denote the 6 o'clock position, as this starting reference point. ${ }^{11,12,16,18}$ Phillipon et al. ${ }^{18}$ proposed the use of the posterior margin of the indirect head of rectus femoris as the 11:30 position, and the center of the Psoas- $U$ (anterior lateral sulcus) as an alternate new reference point at the 3:30 position. Although positions between 12 and 3 o'clock are commonly used for anchor insertion for anterior-superior labral repair, caution is advised during placement of the anchors at the 3 $\mathrm{o}^{\prime}$ clock position, ${ }^{12,13,15,19,20}$ as the acetabular rim becomes narrow at this point and poses a risk for subchondral perforation or psoas tunnel perforation. ${ }^{5,16,19}$

The thickness of the bone around the acetabular rim plays a critical role in determining the drilling angle trajectory. Previous research has suggested that the mean safe angle ranges from $20^{\circ}$ to $30^{\circ}$ across all drill sizes and positions on the acetabulum. ${ }^{1,15}$ However, these findings do not account for any major deformities or pathologies in the hip, the limited working space of the joint, or the large mass of soft tissue that surrounds it. $^{16,21-24}$ Finding a reliable portal that provides safe access into the different compartments of the joint is critical for minimizing iatrogenic damage to the soft or boney tissues of the hip. Although a number of portals have been described previously, no consensus has been reached for either a utilitarian portal for all locations, or specific recommendations for differing portals based on desired rim location. ${ }^{12,15,21,25}$ Determining the optimal portal position and drill trajectory that accounts for anatomical variation in individual anatomy is a modifiable variable in the surgical process that is critical for providing the best postoperative results.

The purpose of this study was to determine the optimal anchor placement and trajectory when repairing acetabular labral tears during hip arthroscopy with the primary focus on the 12 to 3 o'clock positions on the acetabular rim. We hypothesized that an appropriate location would be identified for the optimal trajectory and placement of the suture anchors.

\section{Materials and Methods}

Computed Tomography (CT) scans (GE LightSpeed VCT; General Electric Healthcare, Chalfont, St. Giles, UK; $120 \mathrm{kVp}, 100 \mathrm{~mA}$; slice thickness of $.625 \mathrm{~mm}$ ) of 13 cadaveric lower extremities (pelvis to toes) (3 pairs; mean age $=71.8[10.3]$ ) were used for this study. The scans were imported into 3D Slicer (version 4.10.2: Slicer.org ${ }^{26}$ ), where they were cropped in the sagittal, coronal, and frontal plane, allowing for visualization of the hip and the proximal portion of the femur. The images were thresholded (99-307 HU) to distinguish the bone from the surrounding soft tissues, and two distinct segments were created, isolating the pelvis and the femur geometry. Additional manual editing of the images was performed to ensure all relevant aspects of the bone structures were thresholded appropriately. The segmented and thresholded pelvis was then converted to a 3D geometric model, and smoothing was performed by applying a .5 smoothing factor, and a triangular surface mesh was automatically generated in 3D slicer. The 3D model was then decimated by a factor of .8 to reduce the size of the surface mesh allowing for a smaller file size and easier transfer of the files to subsequent software. The resulting model was converted to an .stl file.

The 12, 1, 2, and $3 \mathrm{o}^{\prime}$ clock positions were identified along the acetabular rim by a fellowship trained orthopaedic surgeon in all models. These positions were confirmed using previous descriptions by Philippon et al., ${ }^{18}$ where the $12 \mathrm{o}^{\prime}$ clock position is opposite to the center of the fovea and transverse acetabular ligament. The psoas- $u$ anteriorly defines the 3:30 position, so the 3 o'clock position was just posterior to this. The 1 and 2 o'clock positions were placed equally between these defined regions, with confirmation that the 2 o'clock position was centered at the base of the AIIS and attachment of the indirect head of the rectus. The confirmed positions were marked with fiducials and were exported to Solidworks (Solidworks Corps 20192020; Dassault Systemes, Vélizy-Villacoublay, France) along with the model stl files (Fig 1). For each of the positions, a plane was generated parallel to the identified point and offset $19.3 \mathrm{~mm}$ into the bone. A $2.7-\mathrm{mm}$ drill diameter and 19.3-mm depth were used for the creation and insertion of virtual cones (Fig 2). These measurements were based off the specifications of a commonly used suture anchor (Smith and Nephew, Andover, MA).

For each position around the acetabulum, a measurement perpendicular to the point at the $19.3-\mathrm{mm}$ drill depth was taken from the extra-articular surface of the acetabulum to the subchondral space. This measurement acted as a reference for the maximal diameter of the cone base inlayed within the acetabulum. This diameter also provided the maximum angle the drill could be placed into the bone without perforating the extra-articular and subchondral surfaces. A single point was embedded at this depth with its location at half the distance between the extra-articular and subchondral surface. From here, truncated cones with the measurements taken from the 12 to 3 o'clock positions were created with the same $2.7-\mathrm{mm}$ diameter top portion, accounting for the drill width and the base, corresponding to the maximal distance from the external to subchondral surface for each specific location. These four cones were labeled according to their corresponding position around the acetabulum and then saved as a part in Solidworks.

A new assembly was created by importing the 3D pelvis part and four cones corresponding to the 12, 1, 2, 


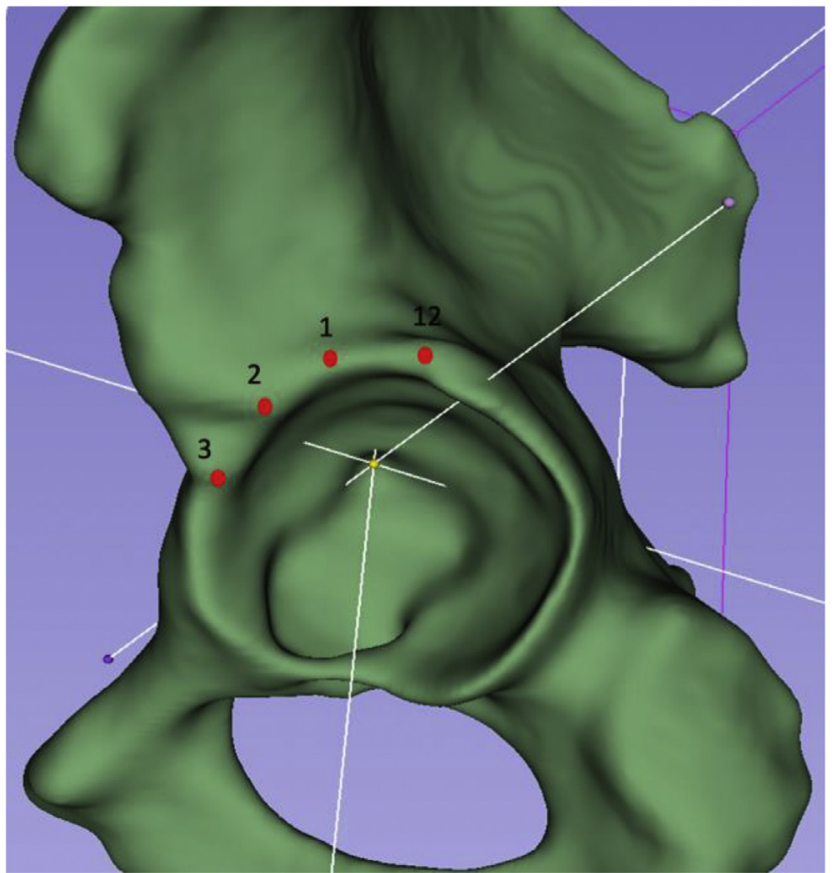

Fig 1. The 3D model of a left acetabulum, showing the 12,1 , 2 and 3 o'clock positions identified by the orthopaedic surgeon.

and 3 o'clock positions for that specific hip model. The cones specifically designed for each location were then attached to the point embedded at the 19.3-mm depth via the center point of the base for that cone using the "mate" feature (Figure 3A). If any part of the cone perforated the bone, it was edited until it was embedded entirely into the bone. After each cone was positioned, mirror images of the cones were extended outward to create an hourglass shape (Fig 3, B and C). It was critical that the edges of the original and mirrored cones remained parallel to each other, as these edges mimic the outer limits of the drill trajectories during surgery.

\section{Data Analysis}

The cones were created to calculate the safe volume of bone and trajectory of the drill for anchor placement. The volume of each of the cones embedded into the bone was quantified by detecting the interference between the hip model and the specific cone at each location. The greater the volume, the safer that location would be for drilling and placing a suture anchor. Secondly, the angle from the center point at the top of each cone to the edges of the base was calculated. The greater the angle, the greater the safety margin the surgeon would have to drill. Third, as each of the cones were extended out toward the surface of the body, calculating the overlap between the bases of the four cones provided an indication of the optimal placement of a portal in order to locate the 12, 1, 2, and 3 o'clock positions. Finally, since the focus of the study was on finding the optimal portal placement for pathologies at the 12 to $3 \mathrm{o}^{\prime}$ clock positions, all data analysis focused on the anterior-superior quadrant of the acetabulum. Comparisons between the portal locations, for each variable were conducted using repeated-measures ANOVA. A Bonferroni adjustment was applied for all post hoc testing and $\alpha$ was set .05 (IBM SPSS v26; Armonk NY).

\section{Results}

\section{Volume}

With respect to the volume of the cones at each position, there was a significant main effect of the anchor location $(P<.001$; ES $=.94$; Power $=1.00)$. There was a significantly larger mean (SD) volume at the $1 \mathrm{o}^{\prime} \mathrm{clock}$ position (4196.2 [1190.2] $\mathrm{mm}^{3}$ compared to all other clock-face positions (Fig 4). There was also statistically less mean (SD) volumes at the 3 o'clock position (629.2 [180.0] $\left.\mathrm{mm}^{3}\right)$ compared to the 12 (1495.9 [411.0] $\left.\mathrm{mm}^{3} ; P<.001\right)$ and 2 o'clock (1375.9 [614.5] $\mathrm{mm}^{3}$; $P=.014)$ positions (Fig 4).

\section{Trajectory Angle}

There was a significant effect of the position of the simulated anchor placement on the trajectory angle $(P<.001 ; \mathrm{ES}=.86 ;$ Power $=1.00)$. The mean $(\mathrm{SD})$

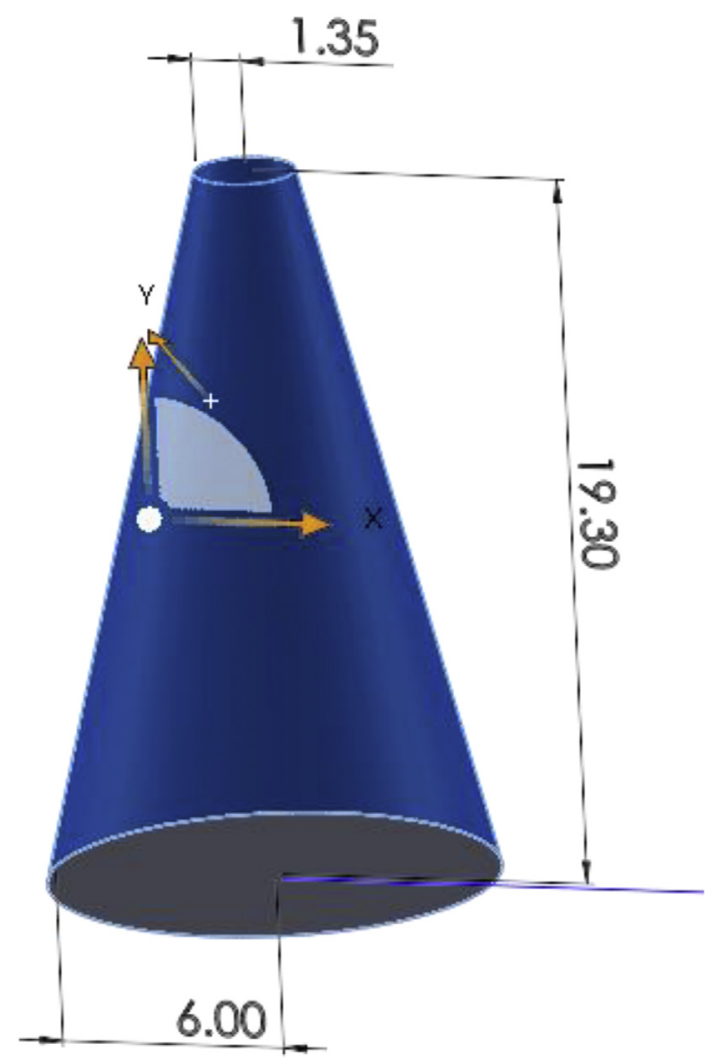

Fig 2. Schematic representation of the cones used to simulate the positioning of suture anchors into the bone of the acetabular rim. 

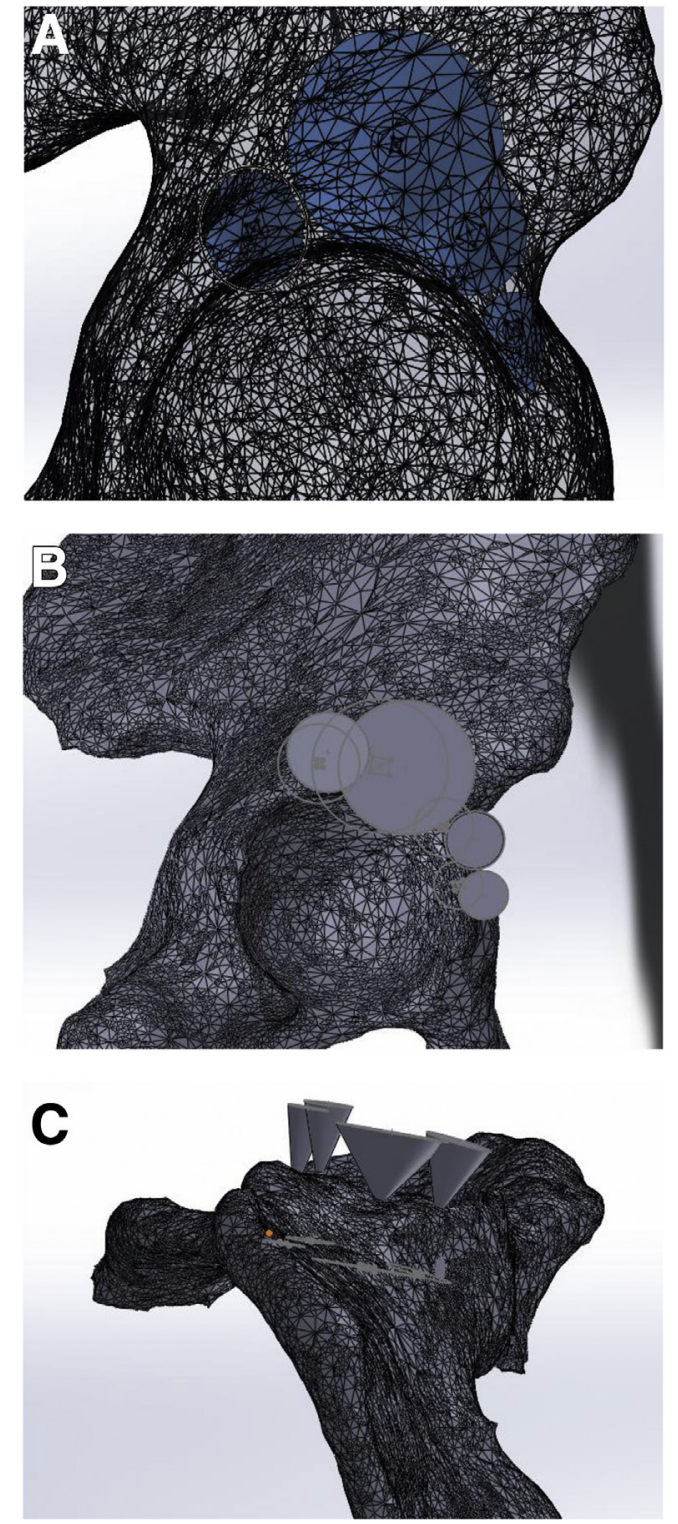

Fig 3. (A) Highlighted in blue are the locations of the cones embedded within the acetabulum at the $4 \mathrm{o}^{\prime}$ clock face positions. Sagittal (B) and axial plane (C) view of the cones extending from within the bone to the superficial surface.

trajectory angle of $32.04[5.05]^{\circ}$ ) at the $1 \mathrm{o}^{\prime}$ clock position was significantly greater compared to all other positions (Fig 5). The shallowest mean [SD] trajectory angle occurred at the $3 \mathrm{o}^{\prime}$ clock position $\left(10.28[2.18]^{\circ}\right)$, and this was significantly different when compared to the 12 $(P<.001)$ and $2 o^{\prime}$ clock $(P=.003)$ positions (Fig 5$)$.

\section{Overlap}

Out of the 13 models, two (specimens 3 and 1) showed no overlap between any of the cones, and four models (specimens 7, 10, 12, and 13) showed only one location where overlap occurred (Table 1). Overall, there was a main effect of the clock face positions on the magnitude of overlap between adjacent positions
$(P=.01 ; \mathrm{ES}=.44$; Power $=.80)$. The least amount of mean (SD) adjacent position overlap occurred between the 2 and 3 o'clock positions $\left(.12[.42] \mathrm{mm}^{3}\right)$, and this was statistically smaller than the overlap between cones at the 12 and $1 \mathrm{o}^{\prime}$ clock positions $\left(214.28\right.$ [251.88] $\mathrm{mm}^{3}$; $P=.029)$ and the 1 and $2 o^{\prime}$ clock positions (139.51 [177.14] $\left.\mathrm{mm}^{3} ; P=.044\right)$ (Table 1).

\section{Discussion}

The main findings of this study were that the bone volumes and trajectory angles were greatest at the 1 o'clock position compared to the other locations around the acetabular rim, suggesting that this is the safest location for anchor insertion, accessible from a wide range of angles. Using a three-dimensional representation of the acetabulum and simulated overlapping cones, it appears unlikely that a utilitarian portal exists to allow safe insertion at all locations, as overlap between all locations was quite limited, and absent in several specimens.

The trajectory angles were the greatest when the simulated suture anchors were placed at the 12 and 1 o'clock positions, providing trajectory angles up to $18.51^{\circ}$ and $32.04^{\circ}$, respectively. These results also indicated that the bone volume is greatest in these two positions, suggesting that surgeons should aim to use this position for the suture anchors, when appropriate, as there is a decreased risk for fragmentation, subchondral perforation, or damage to the joint space. While the 2 o'clock position yielded an average safe trajectory angle similar to the 12 and 1 o'clock

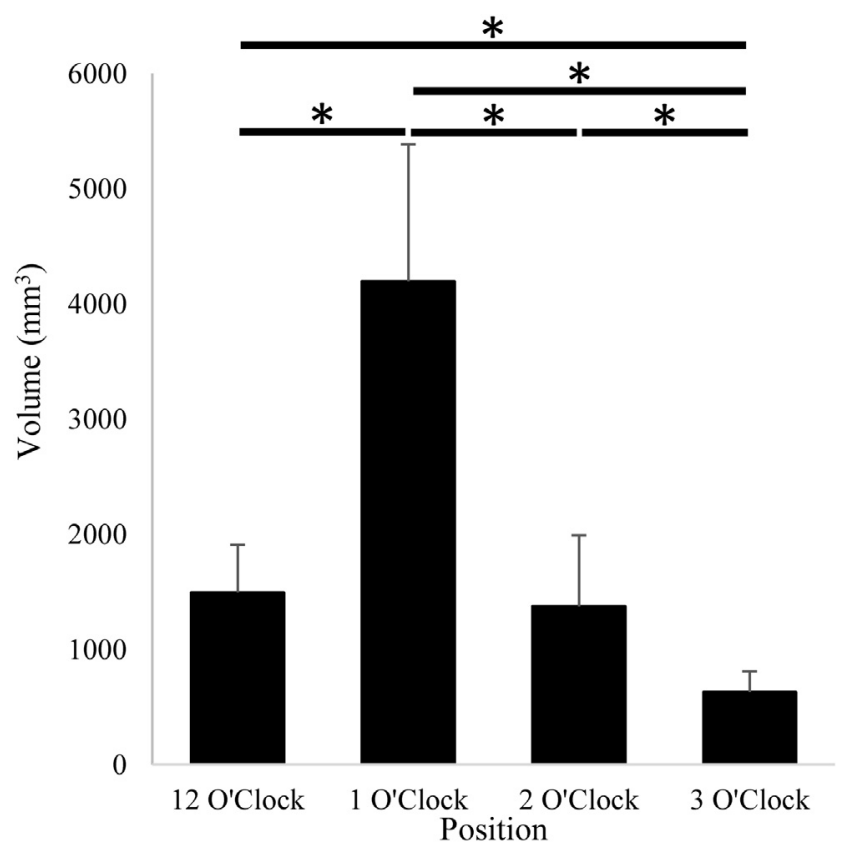

Fig 4. Comparison of the mean (SD) cone volumes embedded in the acetabular rim between the different clock face positions $(* P<.01)$. 


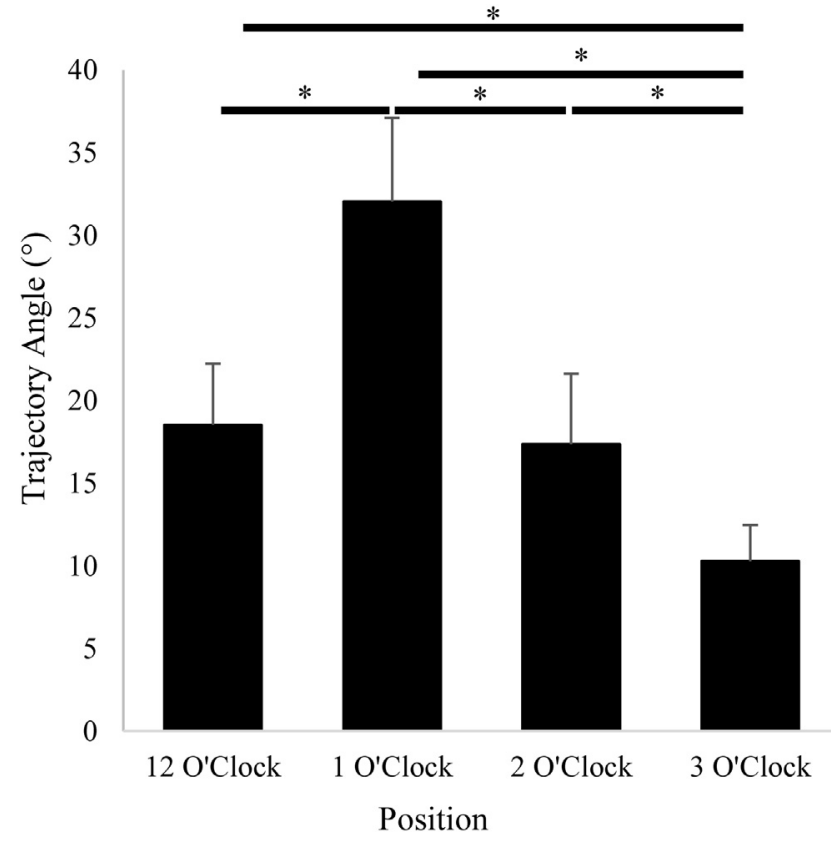

Fig 5. Comparison of the mean (SD) trajectory angles between the different clock face positions $\left({ }^{*} P<.01\right)$.

positions, there was less available bone volume. These results agree with previous investigations that have shown the 2 to 4 o'clock positions to have the greatest risk of perforation. ${ }^{4,13,15,27,28}$ In these previous studies, virtual vectors, physical manipulation with the drill, and the use of custom calipers were used to assess the angles and to create the safety margins.

As previous studies have shown, the narrow portion of bone at the $3 \mathrm{o}^{\prime}$ clock position poses the greatest risk for perforation into the psoas tunnel or intra-articular cartilage. ${ }^{19}$ The current results suggest that caution should be used when using the 2 and 3 o'clock positions, as the margin for error is small and could lead to an undesired perforation with potential clinical implications. Calculating the trajectory angles from the computer-generated cones allowed for the extension of each individual cone from their positions around the acetabulum to the surface of the limb. Identifying the ideal area for portal placement was reliant on two factors: 1) identifying which cones overlapped with each other; and 2) identifying where the extended cones landed with respect to the surface anatomy. As noted earlier, bone volume and safe trajectory angles are greatest at the $1 \mathrm{o}^{\prime}$ clock position and focusing on this location could be key in determining optimal portal placement. Detecting cone interference in Solidworks to evaluate the overlap of cones at the surface of the limb indicated that the cone at the $1 \mathrm{o}^{\prime}$ clock position overlapped the greatest with the 12 and 2 o'clock positions. Caution should be taken when reviewing results from this area of analysis, as some variability was seen in the data across all models. On the basis of the location of overlapping cones, it appears that both the anterolateral and midanterior portals fall within this region and represent safe options for anchor insertion at the studied locations. However, previous literature has reported that distal based portals provide safer trajectory angles into the acetabulum..$^{5,12,16,20}$ It is likely the case, that multiple portals can offer an appropriate approach angle for anchor insertion. However, from a clinical perspective, it appears that use of a single "work-horse" portal for anchor insertion at all locations is not wise, as there were several specimens for which no area overlapped between extrapolated cones. On the basis of our results, the 12 and 1 o'clock locations offer the widest angle and volume of bone for anchor insertion, providing the widest safety margin to avoid perforation, suggesting that portal selection and drill guide trajectory may not be as critical at these locations. However, care must be taken at the 2 and 3 o'clock positions to select a portal that allows direct access with a near-perpendicular approach to the bony surface to stay within the narrower cone of safety to avoid perforation at these locations.

There are some limitations of this study that need to be addressed. The placement of the cones was a manual process, and therefore, this may have contributed a source of variability. However, we attempted to mitigate this variability by having an orthopedic surgeon mark the intended positions of anchor insertion as the location of the cones. Therefore, although this may introduce a source of error, it is similar to the protocol that is used clinically. Another limitation is associated with the use of cadaveric specimens to develop the three-dimensional models. Although, the specimens were inspected to ensure there were no age-related joint issues, bone thickness around the acetabulum may be affected by age. Finally, these results are created with the use of a straight-drill guide, with similar

Table 1. Volume of Overlap $\left(\mathrm{mm}^{3}\right)$ Detected by Interference Between the 4 o'Clock Face Positions for Each Specimen

\begin{tabular}{lccc}
\hline & \multicolumn{3}{c}{ Clock Face Positions } \\
\cline { 2 - 4 } Specimen & 12 and 1 o'clock & 1 and 2 o'clock & 2 and 3 o'clock \\
\hline 1 & 512.65 & 244.62 & 0 \\
2 & 541.91 & 84.92 & 0 \\
3 & 0 & 0 & 0 \\
4 & 111.74 & 10.63 & 0 \\
5 & 101.86 & 132.14 & .08 \\
6 & 552.39 & 356.32 & 0 \\
7 & 0 & 22.17 & 0 \\
8 & 577.71 & 589.82 & 1.52 \\
9 & 13.46 & 0 & 0 \\
10 & 0 & 182.66 & 0 \\
11 & 0 & 0 & 0 \\
12 & 0 & 190.38 & 0 \\
13 & 373.98 & 0 & 0 \\
Mean & 214.28 & 139.51 & $.12^{*}$ \\
SD & 251.88 & 177.14 & .42 \\
\hline
\end{tabular}

*Significantly different than all other adjacent overlaps, $P<.05$. 
interpretation. The use of curved drill guides may improve access at specific locations and perhaps offer more flexibility with portal selection; however, further study is required.

\section{Conclusion}

Trajectory angles and the thickness of bone around the acetabulum were the greatest at the 12 to $1 \mathrm{o}^{\prime}$ clock positions with the $1 \mathrm{o}^{\prime}$ clock position identified as that with the largest trajectory angle for safe anchor insertion.

\section{References}

1. Domb BG, Chen SL, Shapira J, Maldonado DR, Lall AC, Rosinsky PJ. The evolution of hip arthroscopy: What has changed since 2008-A single surgeon's experience. Arthroscopy 2020;36:761-772. doi:10.1016/j.arthro.2019. 10.009.

2. Zhang AL, Feeley BT. Editorial commentary: The rise of hip arthroscopy: Temporary trend or here to stay? Arthroscopy 2018;34:1831-1832. doi:10.1016/j.arthro. 2018.02 .023$.

3. Montgomery SR, Ngo SS, Hobson T, et al. Trends and demographics in hip arthroscopy in the United States. Arthroscopy 2013;29:661-665. doi:10.1016/j.arthro.2012. 11.005.

4. Griffin DW, Kinnard MJ, Formby PM, McCabe MP, Anderson TD. Outcomes of hip arthroscopy in the older adult: A systematic review of the literature. Am J Sports Med 2017;45:1928-1936. doi:10.1177/0363546516667915.

5. Matsuda DK, Bharam S, White BJ, Matsuda NA, Safran M. Anchor-induced chondral damage in the hip. J Hip Preserv Surg 2015;2:56-64. doi:10.1093/jhps/hnv001.

6. Stanton M, Banffy M. Safe angle of anchor insertion for labral repair during hip arthroscopy. Arthroscopy 2016;32: 1793-1797. doi:10.1016/j.arthro.2016.02.013.

7. McCarthy JC, Noble PC, Schuck MR, Wright J, Lee J. The role of labral lesions to development of early degenerative hip disease. Clin Orthop Relat Res 2001;393:25-37. doi:10. 1097/00003086-200112000-00004.

8. Groh MM, Herrera J. A comprehensive review of hip labral tears. Curr Rev Musculoskelet Med 2009;2:105-117. doi:10.1007/s12178-009-9052-9.

9. Blankenbaker DG, De Smet AA, Keene JS, Fine JP. Classification and localization of acetabular labral tears. Skeletal Radiol 2007;36:391-397. doi:10.1007/s00256-0060240-z.

10. Fitzgerald RH. Acetabular labrum tears: Diagnosis and treatment. Clin Orthop Relat Res 1995;(311):60-68.

11. Hernandez JD, McGrath BE. Safe angle for suture anchor insertion during acetabular labral repair. Arthroscopy 2008;24:1390-1394. doi:10.1016/j.arthro.2008.08.007.

12. Degen RM, Poultsides L, Mayer SW, et al. Safety of hip anchor insertion from the midanterior and distal anterolateral portals with a straight drill guide. Am J Sports Med 2017;45:627-635. doi:10.1177/0363546516673836.

13. Lertwanich P, Ejnisman L, Torry MR, Giphart JE, Philippon MJ. Defining a safety margin for labral suture anchor insertion using the acetabular rim angle. Am J Sports Med 2011;39:111S-116S (Supplement 1). doi:10.1177/ 0363546511413746.
14. Nho SJ, Freedman RL, Federer AE, et al. Computed tomographic analysis of curved and straight guides for placement of suture anchors for acetabular labral refixation. Arthroscopy 2013;29:1623-1627. doi:10.1016/j. arthro.2013.07.262.

15. Shah A, Kay J, Memon M, et al. What makes suture anchor use safe in hip arthroscopy? A systematic review of techniques and safety profile. Arthroscopy 2019;35: 1280-1293.el. doi:10.1016/j.arthro.2018.10.118.

16. Dumont GD, Money AJ, Thier ZT. Acetabular subchondral and cortical perforation during labral repair with suture anchors: influence of portal location, curved versus straight drill guides, and drill starting point. Arthroscopy 2019;35:2349-2354. doi:10.1016/j.arthro.2019.02.016.

17. Ilizaliturri VM, Byrd JWT, Sampson TG, et al. A geographic zone method to describe intra-articular pathology in hip arthroscopy: Cadaveric study and preliminary report. Arthroscopy 2008;24:534-539. doi:10. 1016/j.arthro.2007.11.019.

18. Philippon MJ, Michalski MP, Campbell KJ, et al. An anatomical study of the acetabulum with clinical applications to hip arthroscopy. J Bone Jt Surg 2014;96: 1673-1682. doi:10.2106/JBJS.M.01502.

19. Degen RM, O'Sullivan E, Sink EL, Kelly BT. Psoas tunnel perforation-an unreported complication of hip arthroscopy. J Hip Preserv Surg 2015;2:272-279. doi:10.1093/ jhps/hnv043.

20. Foster AD, Ryan J, Ellis T, Flom J. Safe suture anchor insertion for anterior and posterior hip labral repair. $J$ Hip Preserv Surg 2015;2:170-174. doi:10.1093/jhps/hnv027.

21. Aprato A, Giachino M, Masse A. Arthroscopic approach and anatomy of the hip. Muscles Ligaments Tendons $J$ 2016;6:309-316. doi:10.11138/mltj/2016.6.3.309.

22. Kelly BT, Williams RJ, Philippon MJ. Hip arthroscopy: Current indications, treatment options, and management issues. Am J Sports Med 2003;31:1020-1037.

23. Abrams GD, Harris JD, Safran MR. Hip arthroscopy: Portal placement. In: Nho SJ, Leunig M, Larson CM, Bedi A, Kelly BT, eds. Hip arthroscopy and hip joint preservation surgery. New York: Springer, 2015;1-1205. doi:10.1007/ 978-1-4614-6965-0.

24. Shenoy K, Dai AZ, Mahure SA, Kaplan DJ, Capogna B, Youm T. Arthroscopic repair of hip labrum with suture anchors. Arthrosc Tech 2017;6:e2143-e2149. doi:10.1016/j. eats.2017.08.007.

25. Robertson WJ, Kelly BT. The safe zone for hip arthroscopy: A cadaveric assessment of central, peripheral, and lateral compartment portal placement. Arthroscopy 2008;24:1019-1026. doi:10.1016/j.arthro.2008.05.008.

26. Fedorov A, Beichel R, Kalpathy-Cramer J, et al. 3D Slicer as an image computing platform for the Quantitative Imaging Network. Magn Reson Imaging 2012;30: 1323-1341. doi:10.1016/j.mri.2012.05.001.

27. Hewitt J, Guilak F, Glisson R, Vail PT. Regional material properties of the human hip joint capsule ligaments. $J$ Orthop Res 2001;19:359-364. doi:10.1016/S07360266(00)00035-8.

28. Yoo J Il, Ha YC, Hwang SC, et al. Factors associated with the risk of articular surface perforation during anchor placement for arthroscopic acetabular labral repair. Clin Orthop Surg 2017;9:405-412. doi:10.4055/cios.2017.9.4.405. 